\title{
Grounding and ontological dependence
}

\author{
Henrik Rydéhn 1
}

Received: 29 May 2017 / Accepted: 19 May 2018 / Published online: 30 May 2018

(C) The Author(s) 2018

\begin{abstract}
Recent metaphysics has seen a surge of interest in grounding-a relation of non-causal determination underlying a distinctive kind of explanation common in philosophy. In this article, I investigate the connection between grounding and another phenomenon of great interest to metaphysics: ontological dependence. There are interesting parallels between the two phenomena: for example, both are commonly invoked through the use of "dependence" terminology, and there is a great deal of overlap in the motivations typically appealed to when introducing them. I approach the question of the relationship between grounding and ontological dependence through an investigation of their modal connections (or lack thereof). I argue, firstly, that on the common assumption that grounding is factive, it can be shown that no known variety of rigid ontological dependence is either necessary or sufficient for grounding. I also offer some suggestions in support of the claim that this generalizes to every possible form of rigid ontological dependence. I then broaden the discussion by considering a non-factive conception of grounding, as well as by looking at forms of generic (rather than rigid) ontological dependence. I argue that there is at least one form of rigid ontological dependence that is sufficient for non-factive grounding, and that a form of generic dependence may be necessary (but not sufficient) both for factive and non-factive grounding. However, justifying even these fairly weak modal connections between grounding and ontological dependence turns out to require some quite specific and substantive assumptions about the two phenomena that have only rarely been discussed.
\end{abstract}

Henrik Rydéhn

henrik.rydehn@filosofi.uu.se

1 Department of Philosophy, Uppsala University, Box 627, 75126 Uppsala, Sweden 
Keywords Grounding · Dependence · Ontological dependence · Essence · Modality · Fundamentality

\section{Grounding and dependence}

Recent metaphysics has seen a surge of interest in grounding. Grounding is an irreflexive, asymmetric, and transitive relation of non-causal determination. As such, it underlies a particular kind of explanation common in metaphysics and other areas of philosophy. ${ }^{1}$ Consider the following examples:

- Sulla is a bad person because he takes pleasure in the suffering of others.

- The proposition $<$ Snow is white $>$ is true in virtue of snow's being white.

- Cicero is in mental state $M$ because he is in neural state $N$.

Grounding relations have been thought to underlie these and many other explanations. Metaphysicians have recently used grounding ideology to formulate physicalism and truthmaker theory, to define versions of presentism and ontological monism, and to account for metaphysical modality and intrinsicality. ${ }^{2}$ Some have suggested that Plato's Euthyphro question and the Principle of Sufficient Reason should be understood in terms of grounding. ${ }^{3}$ And some have even claimed that, ultimately, all metaphysics concerns questions of grounding. ${ }^{4}$ Whatever the merits of these proposals, grounding is undeniably central to contemporary metaphysics.

Before grounding took center stage in contemporary metaphysical and metametaphysical inquiry, there was similarly intense interest in the notion of ontological dependence. Many of the motivations and inspirations for current research into grounding are strikingly reminiscent of those that animated the literature on ontological dependence. Thus, in an important survey, Fabrice Correia introduces ontological dependence in the following way:

'Ontological dependence' is a term of philosophical jargon which stands for a non-well delineated, rich family of properties and relations which are usually taken to be among the most fundamental ontological properties and relations along with part-whole, exemplification, or again existence.

Most if not all of those who countenance notions of dependence take them to carve reality at its ontological joints. In this connection, ontological dependence is sometimes thought of as marking a certain form of ontological 'non-selfsufficiency'. A dependent object, so the thought goes, is an object whose

\footnotetext{
1 This is slightly contentious. Some philosophers (e.g. Fine 2012; Wilsch 2015) think grounding just is metaphysical explanation, rather than a relation that underlies it. Nothing in the following hinges on this difference.

2 For physicalism, see Dasgupta (2014); for truthmaking, see Schaffer (2008); for presentism, see Baron (2015); for monism, see Schaffer (2010); for modality, see Kment (2014); for intrinsicality, see Witmer et al. (2005).

${ }^{3}$ For the Euthyphro, see Correia and Schnieder (Correia and Schnieder 2012, pp. 2-4). For the PSR, see Dasgupta (2016).

${ }^{4}$ See Schaffer (2009).
} 
ontological profile, e.g. its existence or its being the object that it is, is somehow derivative upon facts of certain sorts - be they facts about other particular objects or not. (2008, p. 1013)

It seems fair to say that most, if not all, of this characterization of ontological dependence could equally well have been found applied to grounding in a contemporary text. And when we look closer at the grounding literature, we find that "dependence" terminology is often applied to grounding as well. For example, Michael J. Clark and David Liggins begin their survey of the grounding literature as follows:

'In virtue of' is a mainstay of philosophical discourse. So are similar expressions, such as 'depends on', 'is prior to', and 'is determined by'. It is reasonable to suppose that these expressions are all used to express claims of non-causal dependence, or - as is becoming the standard term - 'grounding'. (2012, p. 812)

Similarly, Kelly Trogdon starts his introduction to grounding with a list of commonly accepted grounding theses. He then goes on to write

A natural starting point in thinking about grounding is this: is there a single dependence notion corresponding to the various grounding expressions in theses like those listed above? (2013, p. 98)

In his hugely influential article on grounding (titled "Metaphysical Dependence: Grounding and Reduction"), Gideon Rosen points out that

we are often tempted to invoke the idioms of metaphysical dependence, which suggests that we often take ourselves to understand them; and that if we do understand them, we are in a position to frame a number of theses and analyses that appear to be worth discussing. Together these considerations supply us with some reason to believe, and some reason to hope, that the idioms of metaphysical dependence - the grounding idiom, the 'in virtue of' idiom - are clear enough for serious philosophical purposes. (2010, pp. 112-113)

Finally, Jonathan Schaffer, when presenting a novel account of truthmaking, writes

The positive view of truthmaking I would endorse is simple. The key move is to use the notion of dependence (/grounding), instead of swapping it for some modal notion. (2008, p. 311)

Passages like these abound in contemporary work on grounding. Thus, when considering the respective literatures on ontological dependence and on grounding, we are faced with two research programmes that are motivated by strikingly similar considerations, where the target phenomena are both commonly described as forms of dependence. It is thus natural to ask: what is the connection, if any, between grounding and ontological dependence? This will be the central question of the current article. ${ }^{5}$

\footnotetext{
5 Tahko (2015, pp. 104-105) briefly discusses the connection between grounding and ontological dependence and tentatively concludes that "it is advisable to keep the notions apart" (p. 105). Tahko's arguments, however, differ from those presented below.
} 
In the following, I will be approaching the question of the connection between grounding and ontological dependence by investigating a number of modal theses about them. If it turned out that there is some form of ontological dependence that is both necessary and sufficient for grounding, this would be a striking fact. Especially in light of the many similarities between how the two notions are conceived of and characterized, such a modal correlation would at the very least be suggestive of some deeper unity between grounding and ontological dependence. But even a weaker modal connection between the two-i.e. some variety of ontological dependence being either necessary or sufficient for grounding-would constitute an interesting result, since it would allow us to infer certain claims of ontological dependence from grounding claims (or vice versa). And if it were to turn out that ontological dependence is neither necessary nor sufficient for grounding, then this too might be a fruitful discovery, since, presumably, the arguments for that claim would teach us something about the respective natures of the two phenomena.

Though these questions may seem straightforward enough, the issues are somewhat complicated by the fact that both grounding and ontological dependence may be (and have been) conceived of in importantly different ways. These different conceptions generate different specific modal claims to be investigated. In this article, I will argue that if grounding is a factive relation, as on the dominant assumption in the grounding literature, rigid ontological dependence (ontological dependence holding between specific entities) is neither necessary nor sufficient for grounding. I will then show how, by focusing on other conceptions of grounding and/or ontological dependence, it is possible to demonstrate certain connections between the two phenomena. However, justifying even these fairly weak modal connections between grounding and ontological dependence turns out to require some quite specific, substantive and controversial assumptions, some of which have rarely-if ever-even been discussed.

More specifically, I will show (in Sects. 5, 6) that there are both forms of dependence that are sufficient for grounding and forms that are necessary for it. But this will involve focusing on certain forms of generic ontological dependence (where a specific dependent entity does not depend on any other specific entity, but on there being some entity or other satisfying a generic condition), accepting a potentially controversial principle about the modal behavior of grounding, turning to a non-factive conception of grounding, and even considering the idea that grounding (like ontological dependence) comes in a generic variety.

The precise structure of the article is as follows. In Sect. 2, I introduce assumptions and stipulations that I will use in the rest of the discussion. In Sect. 3, I argue that rigid ontological dependence is not necessary for factive grounding, and in Sect. 4 that it is not sufficient for factive grounding either. In Sect. 5, I venture into new territory by considering the relation between non-factive grounding and rigid dependence. Finally, in Sect. 6, I turn to the notion of generic ontological dependence and introduce a (potentially controversial) principle about grounding that would entail a connection between grounding and certain generic dependence claims. I then consider whether the relevant form of generic dependence is necessary or sufficient for either factive or non-factive grounding. 


\section{Preliminaries}

In this section, I will introduce some background assumptions and stipulations that will constrain the discussion that follows. I begin with two assumptions about grounding:

(Assumption 1) Grounding can in principle take relata from any ontological category.

Many philosophers think that grounding holds only between true propositions or obtaining facts. But since ontological dependence can hold between entities of different ontological categories, it is a convenient and simplifying assumption that grounding matches dependence in this respect. ${ }^{6,7}$

My second assumption concerns the formal features of grounding:

(Assumption 2) Grounding necessarily has the following features: irreflexivity, asymmetry, and transitivity. ${ }^{8}$

I will not defend Assumption 2, since that would take us beyond the scope of this article, but it is widely accepted. ${ }^{9}$ One formal feature of grounding that I will not take as fixed is whether it is factive or not. On a factive conception of grounding, it is the case that necessarily, if $y$ is grounded in $x$, then $y$ and $x$ both exist. ${ }^{10}$ As we will see, whether one considers grounding factive or non-factive makes a difference to which connections with ontological dependence one can derive. In Sects. 3 and 4, I will be working with a factive notion of grounding. In Sects. 5 and 6, I will consider non-factive grounding too.

I now proceed to make three terminological stipulations:

(Stipulation 1) The canonical form of a grounding claim is " $y$ is grounded in $x$ " or " $y$ exists in virtue of the existence of $x "$.

The latter formulation highlights that, on the current approach, what gets explained in a grounding explanation is the existence of one entity with reference to the existence of another.

\footnotetext{
6 This is not to say that there are not alternative theses formulated in terms of e.g. propositional grounding that are worth exploring. For example, on such a conception of grounding, one might investigate a parallelism thesis: that necessarily, the proposition that y exists is grounded in the proposition that x exists if, and only if, $y$ ontologically depends on $x$. On that approach to grounding, what gets explained in a grounding explanation is the truth of a proposition, rather than its existence. Though the arguments of this article should, mutatis mutandis, be relevant to that parallelism thesis, I lack the space to consider the issue at length here. See Schnieder (2017) for a fruitful discussion of the relation between what he calls "existential dependence" and "factual dependence". (It should, however, be noted that Schnieder's notion of existential dependence is somewhat narrower than the notion of ontological dependence employed in the current article.) I wish to thank an anonymous referee for comments on this point.

${ }^{7}$ For a treatment that incorporates Assumption 1, see Schaffer (2009).

8 One further feature that is often ascribed to grounding but which I will not assume is that grounding is necessitating in the sense that if $y$ is fully grounded in $x$, then necessarily: if $x$ exists, then $y$ exists. While grounding is generally taken to be necessitating, this has been questioned by Skiles (2015).

${ }^{9}$ For a challenge to irreflexivity, see Jenkins (2011). For a challenge to transitivity, see Schaffer (2012). For a response to both these challenges, see Raven (2013).

${ }^{10}$ Fine (2012, pp. 48-50) discusses factivity and acknowledges both factive and non-factive notions of grounding (though he works with a treatment of grounding as a sentential operator rather than the one used in the current article).
} 
(Stipulation 2) " $<x$ is $F$ (at $t)>$ " denotes the proposition that $x$ is $F($ at $t$ ), and " $[x$ is $F$ (at $t)$ ]" denotes the fact of $x$ 's being $F($ at $t)$.

I take a proposition to be a primary truthbearer and potentially the content of a mental state or declarative sentence. I take a logically atomic fact to be the instantiation of a property by an entity (at a time), or the holding of a relation between entities (at a time). ${ }^{11}$

The third stipulation concerns the relevant sense of "dependence":

(Stipulation 3) Unless otherwise stated, uses of "dependence" denote ontological dependence.

Stipulation 3 allows me to occasionally drop the "ontological" qualifier for stylistic reasons without running the risk of being misunderstood as talking about some other form of dependence of interest in metaphysics, such as e.g. counterfactual dependence. While there are interesting questions about the relation between grounding and counterfactual dependence, these will not concern me here. ${ }^{12}$ As has been noted above, "dependence" terminology is also often used to refer to grounding. It is important to note that Stipulation 3 means that I will diverge from this usage by exclusively using "dependence" for ontological dependence.

At this point, something more needs to be said about ontological dependence. As I've already noted, there is an extensive literature concerning the topic: for important contributions, see Mulligan et al. (1984), Fine (1995), Lowe (1998, ch. 6), Correia (2005), Schnieder (2006), Correia (2008), and Tahko and Lowe (2015). While there is, to the best of my knowledge, no general definition of what makes something a form of ontological dependence, it seems clear that this literature concerns a somewhat unified phenomenon, the different instances of which share certain important characteristics. When talking of dependence, it is thus the phenomenon presented in this literature that I will be concerned with. I will take the notion of ontological dependence as it has been discussed in the relevant literature to constrain the discussion in this article.

One distinction that will become relevant later on and that needs to be flagged already at this point is that there are two major families of ontological dependence notions, distinguished by their logical structure. In forms of rigid ontological dependence, one specific entity $y$ depends on another specific entity $x$. In forms of generic ontological dependence, one specific entity $y$ depends not on any specific entity, but rather on there being some entity or other satisfying a generic condition $\varphi$. The relation between grounding and rigid dependence will be discussed in Sects. 3, 4, and 5, while the relation between grounding and generic dependence is the topic of Sect. 6 .

As anyone familiar with the relevant literature will already know, there are many different forms of ontological dependence even within each of these two major families. ${ }^{13}$ Thus, when I say things like "rigid ontological dependence is not necessary

\footnotetext{
11 On this conception, logically atomic facts are much like the "states of affairs" of Armstrong (1997). I will assume, like Armstrong, that only obtaining facts exist. In what follows, I will usually suppress the reference to a time when naming propositions and facts, since the temporal aspects of propositions and facts will mostly be irrelevant to the issues at hand.

12 See Schaffer (2016) for some discussion of this topic.

13 For comprehensive discussions of various forms of dependence, see Lowe (1998), chapter 6, Correia (2008), and Tahko and Lowe (2015).
} 
for grounding", I should be understood as implicitly quantifying over all the varieties of the relevant form of dependence-e.g. as saying that there is no variety of rigid ontological dependence necessary for grounding.

With these clarifications out of the way, let us move on.

\section{Rigid dependence is not necessary for factive grounding}

In this section, I will argue that there is no variety of rigid ontological dependence that is necessary for factive grounding. I will show this by applying a general argument to various specific forms of rigid dependence. I call this "the Flexibility Argument":

P1. If $y R x$ holds only if $y$ cannot exist unless $x$ exists, then $R$ is not necessary for grounding.

P2. $y R x$ holds only if $y$ cannot exist unless $x$ exists.

$\mathrm{C} 1 . R$ is not necessary for grounding. (P1, P2)

(Although I am currently focusing on factive grounding, we will see in Sect. 5 that the Flexibility Argument applies equally well to non-factive grounding. ${ }^{14}$ ) The idea behind the argument is the following. There are cases where, even though $y$ is grounded in $x, y$ could still exist without $x$. So if a given $R$ holds between $y$ and $x$ only if the former cannot exist without the latter, we can be sure that $R$ is not necessary for grounding. That's P1. In P2, we show, for a specific variety of dependence, that it holds between $y$ and $x$ only if $y$ cannot exist without $x$. It follows that the variety of dependence in question isn't necessary for grounding.

Let me illustrate the Flexibility Argument by applying it to a specific kind of dependence:

(Asymmetrical Rigid Existential Dependence) $y$ depends ARED on $x=\mathrm{df}$. Necessarily, $y$ exists only if $x$ exists, and it is not the case that, necessarily, $x$ exists only if $y$ exists. (Lowe 1998, p. 146$)^{15,16}$

This is the relationship that many have taken to hold between a contingent trope or mode and its bearer: Caesar's cunning cannot exist unless Caesar himself exists, but Caesar could exist without his cunning. If that is right, Caesar's cunning trope depends ARED on Caesar.

\footnotetext{
${ }^{14}$ In Sects. 3 and 4, when I talk without qualification about "grounding", I should be read as referring specifically to factive grounding.

15 See also Correia (2008, p. 1023).

16 The definitions I will be dealing with will all define various predicates expressing different forms of ontological dependence. But I will occasionally need to refer to a form of ontological dependence with the help of a singular term, in which case I will either use an expression like "dependence ARED" (where the subscript corresponds to the subscript of the relevant predicate), or, for variety and increased readability, a name like "asymmetrical rigid existential dependence". Occasionally I will want to talk about the definition of a given form of dependence. In such cases, I will use the capitalized version of the name, for example "Asymmetrical Rigid Existential Dependence" (as in the main text above).
} 
If we plug asymmetrical rigid existential dependence into the Flexibility Argument, P2 plainly turns out true. If $y$ depends ARED on $x$, then necessarily, $y$ exists only if $x$ exists - that's simply the first conjunct of the definiens.

But why believe P1 of the Flexibility Argument? The reason is that there are possible cases of what I'll call flexible grounding. Suppose, for instance, that [Sulla is a morally bad person] is actually grounded in [Sulla takes pleasure in the suffering of others]. So, in the actual world, the former exists in virtue of the existence of the latter. But the former could still have existed without the latter existing. In another possible world, [Sulla is a morally bad person] is grounded in [Sulla is a compulsive liar], and [Sulla takes pleasure in the suffering of others] doesn't even exist. This is a case of flexible grounding. We can contrast this with cases of non-flexible grounding. \{Caesar\} is actually grounded in Caesar, but - unlike in the flexible case-it could have had no other (immediate) ground. In every world where $\{$ Caesar $\}$ exists, it is grounded in Caesar.

You might not accept the specific example of flexible grounding just given. Perhaps you believe the moral badness of a person can only ever be grounded in instantiations of one specific non-moral property $F$ of that person, so that [Sulla is a morally bad person] is in fact non-flexibly grounded in [Sulla is $F$ ]. (What the good-making and bad-making properties of people are is, in the end, an issue for ethics and not metaphysics.) The example is merely intended for illustrative purposes. But it is widely, if not universally, assumed in the grounding literature that there are some cases of flexible grounding. Suppose e.g. that an instantiation of a determinable, like [ $x$ is red], is actually grounded in the instantiation of a determinate- $[x$ is scarlet $]$ - but that it could have been grounded in the instantiation of a different determinate instead-say $\left[x\right.$ is crimson]. ${ }^{17}$ Or consider an existential generalization [There is an $F$ ], where some actual $F$ s are contingent existents and there could have been other $F$ s. Almost every friend of grounding is thus, by recognizing examples like these, committed to there being some possible cases of flexible grounding. I am simply relying on this shared commitment. (And this commitment is not theoretically idle. When discussing an objection below, I will outline one of the uses of recognizing modally flexible grounding. ${ }^{18}$ )

Since there can be flexible grounding, any $R$ which holds between two entities $y$ and $x$ only if $y$ cannot exist without $x$ fails to be necessary for $y$ 's being grounded in $x$. And since we have seen that $\mathrm{P} 2$ is true when we plug in dependence ARED $_{\text {, we }}$ have thereby shown that asymmetrical rigid existential dependence is not necessary for grounding.

\footnotetext{
17 For an introduction to the determinate/determinable distinction and some philosophical issues surrounding it, see Wilson (2017).

18 What if Lewisian counterpart theory, according to which each individual is worldbound, is true? Such a view is still compatible with flexible grounding. The counterpart theorist can perfectly well recognize cases where an individual $y$ is actually grounded in some entity $x$, but where $y$ could have had some other ground-she will simply provide an account of this in terms of there being some $y$-counterpart that is grounded in something that is not an $x$-counterpart. For a classical statement and defense of counterpart theory, see Lewis (1986, pp. 192-263). I wish to thank an anonymous referee for raising this issue.
} 
P1 is true regardless of which variety of rigid dependence is inserted into the argument. It remains to show for different specific rigid dependence notions that each of them renders P2 true. I will proceed to this task after answering an objection to P1.

The objection is that flexible grounding isn't really possible when we individuate the relata of grounding finely enough. According to the objection, possible worlds should enter into facts as constituents. So it's not that [Sulla is a morally bad person] is grounded in [Sulla takes pleasure in the suffering of others]; rather, grounding holds between [Sulla is a morally bad person in $w_{1}$ ] and [Sulla takes pleasure in the suffering of others in $w_{1}$ ]. And when the relata are individuated this finely, flexible grounding is trivially ruled out. If any fact involving Sulla's badness is grounded at another possible world, it will be a fact like [Sulla is a morally bad person in $w_{2}$ ]. But since the relevant grounded facts in $w_{1}$ and $w_{2}$ differ in one of their constituents, they cannot be identical. Thus, applied consistently, this approach would rule out the possibility of any flexible grounding, thereby removing the motivation for P1.

My response to the objection is that we should reject this way of individuating the relata. Firstly, it is ad hoc. The relata of grounding typically aren't individuated this finely. We'd need some independent motivation for doing so beyond avoiding the Flexibility Argument, and I can't see that there is one.

Secondly, the suggested approach has problematic consequences. Important applications of grounding presuppose that it can be flexible. For example, due to considerations of multiple realizability, one might not want to identify [Cicero is in mental state $M$ ] with [Cicero is in neural state $N_{1}$ ]. If the facts were identical, the former couldn't exist without the latter-since nothing can exist without itself. But if the former is multiply realizable, then it could exist without the latter. Therefore, those who hold that mental states are multiply realizable do not identify [Cicero is in mental state $M$ ] with [Cicero is in neural state $N_{1}$ ], but might instead hold that the former is grounded in the latter. But grounding can only be employed to formulate a view like this if we allow for the possibility of flexible grounding. If this is ruled out, as per the objection, then grounding will be no more modally flexible than identity. So I conclude that the objection fails: it is both undermotivated and would rule out important applications of grounding.

Having thus defended the Flexibility Argument, I will now argue, for various forms of rigid dependence, that each of them renders $\mathrm{P} 2$ true when plugged into the argument. Given that P1 is true (as I've argued above), this shows that none of these dependences are necessary for grounding. I will start with explanatory existential dependence:

(Explanatory Existential Dependence) $y$ depends EED $_{\text {on }} x=\mathrm{df}$. Necessarily:

(i) if $y$ exists, then $x$ exists; and

(ii) if $y$ exists and $x$ exists, then $y$ exists at least partially because $x$ exists. ${ }^{19}$

According to Lowe, explanatory existential dependence holds between the extended event that is Socrates' life and Socrates himself. Even though the two exist in all the

19 Adapted from Lowe (1998, p. 145). I have unpacked Lowe's original definition somewhat to make it clearer; I believe my adaptation is true to his intentions. 
same worlds, there is (on Lowe's view) an explanatory asymmetry between them: the event exists at least partially because the substance does, but not vice versa.

Clause (i) guarantees that when we plug explanatory existential dependence into the Flexibility Argument, $\mathrm{P} 2$ is true: if $y$ depends $\mathrm{EED}_{\mathrm{E}}$ on $x$, then $y$ cannot exist without $x$. So explanatory existential dependence isn't necessary for grounding.

A rather similar notion is defined by Fabrice Correia:

(Simple Foundation) $y$ dependssF on $x=$ df. Necessarily:

if $y$ exists, then either $y$ is at least partially grounded in $x$, or $y$ is at least partially grounded in some fact about $x .^{20}$

Simple foundation is in one way more flexible than explanatory existential dependence. It may e.g. be that [Cicero is feeling sad] dependssF on Cicero's brain without being grounded in it, as long as [Cicero is feeling sad] is grounded in some fact about Cicero's brain-such as e.g. [Cicero's brain is in neural state $N$ ].

But Correia imposes an important requirement on any fact satisfying the second disjunct of the definiens. The property instantiated by an entity in the fact must be existence-entailing in the sense that a thing cannot instantiate the property without existing (Correia 2005, p. 66). It is therefore clear that Simple Foundation renders P2 true. Suppose $y$ depends $\mathrm{SF}_{\mathrm{SF}}$ on $x$. Then it follows that in every world where $y$ exists, $y$ is at least partially grounded directly in $x$, or $y$ is at least partially grounded in some fact about $x$, where that fact cannot exist without $x$ existing. Either way, $x$ must exist for $y$ to exist. So simple foundation is not necessary for grounding.

Let us move on to varieties of essential dependence-forms of dependence defined with the help of essentialist ideology. I will here be concerned with the kind of robust essentialism associated with philosophers like Kit Fine and E. J. Lowe. ${ }^{21}$ On this approach, what is essential to a thing concerns the identity of that thing, what it is to be that thing, and thereby goes beyond what is necessarily true of it.

Consider first essential existential dependence:

(Essential Existential Dependence) $y$ depends $s_{\mathrm{EDE}}$ on $x=\mathrm{df}$. It is part of the essence of $y$ that $y$ exists only if $x$ exists. (Tahko and Lowe $2015, \$ 4.3)$

This is the form of dependence that is typically taken to hold between $\{$ Caesar\} and Caesar. Though they exist in all the same possible worlds, it is part of the singleton's essence that it exists only if the man does. The essence of the man, however, does not involve the set in any way.

Plugging essential existential dependence into the Flexibility Argument renders P2 true. According to the robust kind of essentialism we're considering, if a proposition $\varphi$ is part of the essence of a thing, $\varphi$ is metaphysically necessary (Fine 1994, p. 4; Correia 2005, pp. 26-27). ${ }^{22}$ So if $y$ depends $\mathrm{EDE}$ on $x$, then necessarily, $y$ exists only

\footnotetext{
20 Adapted from Correia (2005, p. 66). Since Correia works with a different formal framework for grounding, I have had to reformulate his definition somewhat. I believe my adaptation is faithful to the spirit of the original definition.

21 For the locus classicus of this approach in contemporary analytic philosophy, see Fine (1994).

22 Sometimes essentialists commit only to the weaker principle that if it lies in the essence of $x$ that $\varphi$, then it is metaphysically necessary that if $x$ exists, then $\varphi$. But the choice between the stronger and the weaker
} 
if $x$ exists. That is precisely what is needed for $\mathrm{P} 2$ to be true. So essential existential dependence is not necessary for grounding.

Finally, I will go on to consider two similar forms of essential dependence: (Identity-Dependence)

$y$ depends $_{\mathrm{ID}}$ on $x=\mathrm{df}$. There is a two-place predicate " $F$ " such that it is part of the essence of $y$ that $y$ is related by $F$ to $x$. (Tahko and Lowe $2015, \S 4.2$ )

(Definitional Essential Dependence) $y$ depends DED $_{\text {on }} x=\mathrm{df} . x$ is a constituent of a proposition that is true in virtue of the identity of $y$. (Fine 1995, p. 275)

Definitional Essential Dependence requires some unpacking. Fine's approach to dependence is rooted in his view of essence as closely tied to real definition. When a real definition is successful, it expresses the identity of the thing defined, and any part of the definition is "true in virtue of the identity" of the thing. So we might say that $y$ depends $\mathrm{DED}_{\mathrm{D}}$ on $x$ if and only if $x$ is a constituent of a proposition that is part of the real definition of $y$.

The authors behind these definitions of dependence clearly intend that if $y$ depends on $x$ in the relevant sense, then $y$ cannot exist without $x$ :

If $x$ [depends ID] upon $y$, then, necessarily, $x$ exists only if $y$ exists. [...] [S] urely, $x$ cannot exist unless everything upon which $x$ 's identity depends also exists. (Tahko and Lowe 2015, §4.2)

What is maintained is that, given that one object is ineliminably involved in the nature of another, then it is not compatible with the nature of the second that it should exist without the first. (Fine 1995, p. 279)

If this is right, the varieties of dependence in question will render P2 of the Flexibility Argument true. If $y$ depends ID $_{\text {or depends }}$ of on $x, y$ cannot exist unless $x$ exists. Consequently, neither of identity-dependence or definitional essential dependence is necessary for grounding.

I've now surveyed a number of central kinds of rigid dependence and argued that none of them are necessary for grounding. They all render the Flexibility Argument sound. And as far as I know, there is no form of rigid dependence that does not. That no extant form of rigid ontological dependence is necessary for grounding is a noteworthy and interesting result in and of itself.

But even given this result, one might wonder if there could be some form of rigid ontological dependence, hitherto undiscovered, that avoids the Flexibility Argument. This question is not easy to answer, since there is no settled account of what makes something a form of ontological dependence. However, we have seen that all the forms

Footnote 22 continued

principle makes no difference to the current argument. The weaker principle together with the assumption that $y$ depends $\mathrm{EDE}$ on $x$ entails that it is metaphysically necessary that if $y$ exists, $y$ exists only if $x$ exists. But if this is metaphysically necessary, then it follows that $y$ cannot exist unless $x$ exists. And that is what is needed for P2 to be true. Thus, the Flexibility Argument applies to essential existential dependence even on the weaker principle about the connection between essence and necessity. I would like to thank an anonymous referee for encouraging me to discuss the weaker essentialist principle. 
of rigid dependence surveyed here entail that the dependent entity cannot exist without the entity on which it depends (and the same seems to go for the varieties that have not shown up in the preceding discussion). It would be curious if this turned out to be a sheer coincidence.

One thing which would explain this (and thus render it non-coincidental) would be if there were some use of the notion of ontological dependence that enforced this behavior as a necessary condition for being a form of dependence. I will now tentatively suggest a candidate for such a use. It is a plausible necessary condition for $R$ 's counting as a form of rigid ontological dependence that $y R x$ entails that $y$ cannot exist without $x$. And the suggestion is that this condition is due to a certain functional role that dependence notions can play in ontological inquiry: to generate a kind of hypothetical ontological commitment.

One way to think about ontological dependence theses is that they constrain the form of an acceptable ontology. Why, for example, does it matter whether tropes are ontologically dependent on the objects they characterize? One of the reasons is that if so, this generates a kind of hypothetical commitment: if you postulate tropes, you must postulate objects that they characterize as well. Whether we should accept a commitment of that kind is an important issue in ontology - and one on which friends of tropes can disagree. And for rigid dependence notions, the hypothetical commitment will be to the existence of a specific entity. So it makes a lot of sense of discussions of ontological dependence if we assume that something counts as rigid dependence only if a dependent entity cannot exist without that on which it depends.

If this proposal is right, it would not only explain why every extant form of rigid dependence has been subject to the Flexibility Argument but would also render every possible form of rigid dependence equally susceptible to the argument. Of course, what has been said here in defense of the proposal has been highly tentative and inconclusive. But the difficulties with saying anything general on this point are a consequence of our imperfect understanding of the nature of ontological dependence. Anyone who claims to have identified a form of rigid ontological dependence that is not susceptible to the Flexibility Argument faces the opposite challenge of explaining why the alleged counterexample should count as belonging to the same family as the notions discussed above. With this said, we will have to move on.

\section{Rigid dependence is not sufficient for factive grounding}

In this section, I will argue that there is no variety of rigid ontological dependence that is sufficient for grounding, with grounding taken as factive. I will start out by clarifying what I mean by a form of dependence being sufficient for grounding. By committing to Stipulation 1 in Sect. 2 above, I have taken the canonical form of a grounding claim to be " $y$ is grounded in $x$ ". This treats grounding as a relational predicate that applies to specific entities $y$ and $x$. So for a specific form of rigid dependence (say "dependence ${ }_{\mathrm{R}}$ ") to be sufficient for grounding would simply be for $y$ 's depending $g_{R}$ on $x$ at a world $w$ to entail that $y$ is grounded in $x$ at $w$. For generic forms of dependence (which will be discussed at length in Sect. 6), the situation is slightly less straightforward. Unlike rigid dependence and grounding, generic dependence is 
not expressed by a two-place predicate applying to two specific named entities $y$ and $x$ where the first is the dependent/grounded entity and the second the dependee/ground. A generic dependence claim is rather of the form " $x$ depends $_{\mathrm{G}}$ on $F \mathrm{~s}$ ". But we can relax the requirement for sufficiency in the case of generic dependence somewhat. I will take $y$ 's depending ${ }_{X}$ on $F$ s to be sufficient for grounding just in case $y$ 's depending on $F \mathrm{~s}$ at world $w$ entails the truth of some specific claim of the form " $y$ is grounded in $x$ " at $w$. (We will return to this criterion at some length in Sect. 6.)

With this clarification out of the way, we can turn to the question of whether rigid dependence is sufficient for grounding. In this section, I will take grounding to be necessarily factive in the sense that if $y$ is grounded in $x$, then $y$ and $x$ both exist. This feature is typically taken to flow from the connection between grounding and explanation. If $y$ is grounded in $x$, the existence of $y$ is non-causally explained by the existence of $x$. And if $y$ 's existence is explained with reference to $x$ 's existence, both $y$ and $x$ must indeed exist. (I will discuss non-factive grounding in Sect. 5.)

Consider again explanatory existential dependence:

(Explanatory Existential Dependence) $y$ depends EED $_{\text {on }} x=\mathrm{df}$. Necessarily:

(i) if $y$ exists, then $x$ exists; and

(ii) if $y$ exists and $x$ exists, then $y$ exists at least partially because $x$ exists.

Explanatory existential dependence is not sufficient for factive grounding. To see this, suppose that $e$ is some entity that does not actually exist, but which could have existed- $e$ is a contingently non-existent entity. Suppose further that the familiar assumptions about the metaphysics of sets hold: whenever $e$ exists, its singleton $\{e\}$ exists too, and is grounded in $e$-and that is the only way $\{e\}$ can exist. It follows that $\{e\}$ depends $\mathrm{EED}_{\mathrm{E}}$ on $e$-necessarily, if $\{e\}$ exists, $e$ exists, and necessarily, if both exist, then $\{e\}$ exists at least partially because e exists. And note that it follows that $\{e\}$ depends $\mathrm{EED}_{\mathrm{E}}$ on $e$ even in the actual world, since, in the actual world, $\{e\}$ and $e$ jointly satisfy the definition of explanatory existential dependence. But we were assuming that neither $e$ nor $\{e\}$ exists in the actual world. So by the factivity of grounding, $\{e\}$ isn't grounded in $e$ in the actual world. We have a case of explanatory existential dependence without factive grounding: so the former isn't sufficient for the latter.

Another way of seeing this point is to consider that if explanatory existential dependence holds between two entities in any world, then it holds between those entities in all possible worlds. But factive grounding need not behave like this: if a grounded entity exists only contingently, then it will fail to be grounded in any world where it doesn't exist.

Now consider again simple foundation:

(Simple Foundation) $y$ dependssF on $x=$ df. Necessarily:

if $y$ exists, then either $y$ is at least partially grounded in $x$, or $y$ is at least partially grounded in some fact about $x$.

Simple foundation is not sufficient for factive grounding either. The contingently nonexisting entities $\{e\}$ and $e$ do not exist in the actual world, so the former isn't actually grounded in the latter. Still, $\{e\}$ depends $\mathrm{SF}$ on $e$ in the actual world, since it is actually true that, necessarily, if $\{e\}$ exists, then it is at least partially grounded in $e$. So the actual world presents us with a case of simple foundation without (factive) grounding. 
Finally, let us consider our three varieties of essential dependence:

(Essential Existential Dependence) $y$ depends $\operatorname{EDE}_{\mathrm{E}}$ on $x=\mathrm{df}$. It is part of the essence of $y$ that $y$ only exists if $x$ exists.

(Identity-Dependence)

$y$ depends $\mathrm{ID}$ on $x=\mathrm{df}$. There is a two-place predicate " $F$ " such that it is part of the essence of $y$ that $y$ is related by $F$ to $x$.

(Definitional Essential Dependence) $y$ depends DED $_{\text {on }} x=\mathrm{df} . x$ is a constituent of a proposition that is true in virtue of the identity of $y$.

None of these are sufficient for factive grounding, for the same reason. Essences are, according to robust essentialism, invariant across all worlds (Correia 2005, p. 27). So if $\varphi$ is part of the essence of $y$ in one world, then $\varphi$ is part of the essence of $y$ in every possible world. It follows that if $y$ and $x$ satisfy one of our three forms of essential dependence in any possible world, they do so in every possible world-even ones where $y$ and $x$ do not exist. But $y$ will fail to be grounded in $x$ in any world where the two don't exist. Thus, none of these forms of essential dependence are sufficient for (factive) grounding.

I have argued that none of these central kinds of dependence are sufficient for factive grounding. I know of no variety of rigid dependence that is not subject to the same kind of considerations. But before turning to the question whether this result can confidently be generalized to all possible forms of dependence, I will now pause to consider an objection.

The objection holds that the argument presupposes an implausible thesis. My argument relied on the claim that one thing can depend on another even when the two do not exist. But, the objection goes, relations hold only between existent entities and ontological dependence is a relation, so that claim is false.

This objection is not convincing. Insofar as relations hold only between existents, I am happy to deny that ontological dependence is a relation. For illustration, consider again simple foundation:

(Simple Foundation) $y$ dependssF on $x=$ df. Necessarily:

if $y$ exists, then either $y$ is at least partially grounded in $x$, or $y$ is at least partially grounded in some fact about $x$.

The left-hand side of the definition takes the form of a relational expression. But if we want to know if the notion defined expresses a relation, we should look to the definiens rather than the definiendum. And, clearly, the definiens (the whole necessitated conditional) can be satisfied (at a world) without any relation holding between entities existing at that world. The definiens states that every world where $y$ exists is also a world where $y$ is at least partially grounded in $x$ or some (existence-entailing) fact about $x$. Now suppose that $w_{1}$ and $w_{2}$ are the only possible worlds and that \{Pegasus and Pegasus both exist at $w_{1}$ but neither exists at $w_{2}$. At $w_{1},\{$ Pegasus $\}$ and Pegasus are related by grounding - and that is fine, since they both exist at $w_{1}$. At $w_{2}$, neither \{Pegasus $\}$ nor Pegasus exists, so no relations hold between them. Since $w_{1}$ and $w_{2}$ are all the possible worlds, this suffices to guarantee that at every world where $\{$ Pegasus $\}$ exists, $\{$ Pegasus $\}$ is either at least partially grounded in Pegasus, or at least 
partially grounded in some fact about Pegasus. \{Pegasus\}'s depending SF $_{\text {on }}$ Pegasus amounts to no more than the full space of possible worlds being structured in that way. And the full space of possible worlds is, of course, structured in that way even at $w_{2}$, where \{Pegasus $\}$ and Pegasus do not exist, without this requiring any relations to hold between $\{$ Pegasus $\}$ and Pegasus at $w_{2}$. The objection fails. ${ }^{23}$

I thus take the preceding to show that none of the forms of rigid ontological dependence discussed above are sufficient for (factive) grounding. And I know of no other variety of rigid dependence that is not subject to the same argument. But, just as with the argument of the preceding section, we can ask how far this result can be extended.

More specifically, we might wonder why we couldn't get a form of dependence sufficient for grounding by simply adding a requirement of factivity. We could, for example, define a new notion on the basis of Simple Foundation:

(Simple Foundation*) $y$ depends SF* $^{*}$ on $x=\mathrm{df}$.

(i) $y$ exists; and

(ii) necessarily: if $y$ exists, then either $y$ is at least partially grounded in $x$, or $y$ is at least partially grounded in some fact about $x$.

The factivity of grounding would not give rise to counterexamples to the sufficiency of simple foundation*. It follows from the definition that if $y$ depends $\mathrm{SF}^{*}$ on $x$, then $y$ and $x$ both exist, so, in this respect at least, simple foundation* is on a par with factive grounding.

At this point, we once again face the difficult question of what it takes to count as a form of (rigid) ontological dependence. We've seen that every dependence notion $R$ surveyed above satisfied the condition that if $R$ applies to $y$ and $x$ in any possible world, it applies to them in all worlds. Again, it would seem surprising that this is the case, unless the condition was connected to some important common feature of the family of ontological dependence notions. By contrast, if that condition were a necessary condition for counting as a variety of rigid ontological dependence, it would be entirely unsurprising that all the extant forms of rigid dependence in the literature uniformly behave in this way, despite varying along a number of other dimensions. And the condition in question is no longer satisfied when we introduce a requirement of factivity into the definition.

Since we have no clear account of what makes something a form of ontological dependence, I suggest again that the best we can do is turn to the possible uses of dependence notions. And one interesting use that would unify the varieties of ontological dependence under the proposed necessary condition is that they could all figure in simple and straightforward explanations of non-existence. For example, if someone were to ask why \{Pegasus $\}$ does not exist, an intuitively correct answer would be that it is because \{Pegasus \} depends ontologically on Pegasus, and Pegasus doesn't exist. But a factive notion like simple foundation* (defined above) cannot be used

\footnotetext{
23 The objection makes the most sense against the background of a sparse conception of properties and relations, on which there need not necessarily correspond any property or relation to a meaningful predicate. On an abundant conception of properties and relations, it is perhaps harder to deny that ontological dependence is a relation. But on that conception, the claim that relations hold only between existent entities looks much less plausible too. I wish to thank an anonymous referee for raising this point.
} 
like this: if $\{$ Pegasus $\}$ depends $\mathrm{SF}^{*}$ on Pegasus, then ipso facto \{Pegasus exists. So we cannot achieve this straightforward explanation on the basis of simple foundation* and similar notions. The proposed necessary condition on ontological dependence that rules out notions like simple foundation* would thus not be arbitrary or unmotivated, but connects with a natural and fruitful use dependence notions can be put to in our theorizing. ${ }^{24,25}$

A possible response is that factive dependence notions too can play this explanatory role. In a world where Pegasus does not exist, it might not be true that \{Pegasus\} depends on Pegasus, the response goes. But it will still be true that had Pegasus existed, $\{$ Pegasus $\}$ would have depended $\mathrm{SF}_{*}$ on Pegasus. This, together with the fact that Pegasus does not exist explains why $\{$ Pegasus $\}$ does not exist.

This response is not convincing. The explanatory strategy is unnecessarily roundabout since, on reflection, even the respondent has a perfectly good explanation available that is based wholly in the actual world. She too is in fact committed to P Pega-

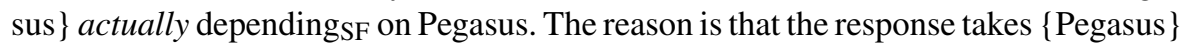
to depend $\mathrm{SF}^{*}$ on Pegasus in the closest possible world where Pegasus exists-call it " $w$ *". Now consider that, by the definition of simple foundation*, this amounts to the following being true at $w^{*}$ : (i) \{Pegasus $\}$ exists; (ii) necessarily: if \{Pegasus $\}$ exists, then either \{Pegasus\} is at least partially grounded in Pegasus, or \{Pegasus $\}$ is at least partially grounded in some fact about Pegasus. (ii) being true at $w^{*}$ thus amounts to

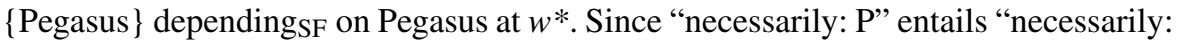
necessarily: P" when dealing with metaphysical necessity, it follows that (ii) is true not only at $w^{*}$ but at all worlds-including the actual world. ${ }^{26}$ So the respondent too is committed to non-factive dependence between \{Pegasus $\}$ and Pegasus in the actual world. But then it makes little sense to explain why \{Pegasus\} doesn't exist in the actual world with reference to a factive dependence that only holds counterfactually. Even the respondent accepts that there is a variety of dependence that holds in the actual world which is sufficient to prevent \{Pegasus\} from existing without Pegasus. That kind of dependence makes for a better explanation of \{Pegasus\}'s non-existence. The response thus fails.

\footnotetext{
${ }^{24}$ To be clear, when I say that the necessary condition in question "rules out" notions such as simple foundation*, this means that it rules them out from counting as forms of ontological dependence. I do not wish to suggest that such notions are incoherent, uninteresting, or never exemplified. I wish to thank an anonymous referee for pushing me to clarify the argument on several points.

25 It might be thought that this explanatory claim can be handled with reference to simple foundation*. After all, the modal dependence claim that \{Pegasus $\}$ cannot exist unless Pegasus exists is entailed by the claim that $\{$ Pegasus $\}$ depends $\mathrm{SF}^{*}$ on Pegasus. And that modal dependence can be part of an explanation of \{Pegasus\}'s non-existence. But while this is all true, it does not help the case against my proposed necessary condition. The modal dependence entailed by simple foundation* is, unlike simple foundation* itself, non-factive and does obey the necessary condition on ontological dependence that I have proposed. And it is the modal dependence in question, and not the factive notion of simple foundation* as a whole, that does the relevant explanatory work in the suggestion here outlined. (Since, by design, \{Pegasus $\}$ doesn't depend $\mathrm{SF}^{*}$ on Pegasus in a world where \{Pegasus\} doesn't exist, and since only what is true can explain, simple foundation* is not itself available to do any explanatory work at the world in question.) I would like to thank an anonymous referee for suggesting I explore this alternative.

${ }^{26}$ I am thus here assuming a system of modal logic at least as strong as S4. This assumption is standardly made for metaphysical modality.
} 
I have here outlined a possible necessary condition on (rigid) ontological dependence that would entail that the argument of this section generalizes to all possible forms of rigid dependence. But it must be admitted that-perhaps to an even higher degree than with the generalization of the previous section's argument-this is all fairly speculative, since the limits of ontological dependence are presently not well explored. A principled alternative account of how the ontological dependence family is unified that allows for both factive and non-factive varieties of ontological dependence might certainly constitute an interesting contribution to our understanding of the notion. For now, I will have to move on.

\section{Non-factive grounding and rigid dependence}

In Sects. 3 and 4, I argued that rigid dependence is neither necessary nor sufficient for factive grounding. If my arguments are correct, they provide us with an interesting part of the story about the relation between grounding and ontological dependence. The preceding discussion has, however, been limited in a number of ways. Firstly, I have been working with a factive conception of grounding. Secondly, I have so far only considered rigid varieties of dependence. In this section, I wish to widen the scope of the discussion by considering what a non-factive conception of grounding would imply for the connection with rigid dependence. In the following section, I will introduce generic forms of dependence, and consider how a seemingly plausible principle about the modal behavior of grounding would impact the issues at hand.

I turn first to non-factive grounding. As I noted in Sect. 2 above, the vast majority of writers on grounding have been committed to the factivity of grounding-either by outright accepting factivity or by committing to other claims about grounding that arguably entail that it is factive. However, recent work on grounding has seen some notable exceptions to this (e.g. Fine 2012; Correia 2014; Litland 2017).

On a non-factive conception of grounding, $y$ can be grounded in $x$ even when $y$ and $x$ do not exist. ${ }^{27}$ To help fix the idea of non-factive grounding, we can follow a suggestion considered by Fine (2012, p. 49) that $y$ is non-factively grounded in $x$ if, and only if, $y$ is possibly factively grounded in $x .^{28}$ We can then ask how employing a non-factive notion of grounding would affect the arguments laid out in previous sections.

I will start by considering whether it is plausible that rigid ontological dependence is necessary for non-factive grounding. And here it seems that the same argument employed in Sect. 3 still applies: since there can be flexible grounding, we can have cases of non-factive grounding without rigid ontological dependence.

\footnotetext{
27 It should be noted that this is not exactly how Fine (2012), Correia (2014) and Litland (2017) cash out non-factive grounding, since they do not treat grounding in terms of a relational predicate applying to entities of arbitrary ontological categories.

28 To accept this biconditional is not to assume that non-factive grounding should be defined in terms of factive grounding, or that factive grounding is metaphysically prior to non-factive grounding. Fine (2012, p. 49) argues that the two notions are in principle interdefineable. Fine also surveys some considerations which he takes to "strongly suggest that the factive notion is the more fundamental notion" (p. 49). I will not take any stance on these issues here.
} 
To see this, suppose that metaphysical space is exhausted by the three possible worlds $w_{1}, w_{2}$, and $w_{3}$. At $w_{1}$, [Sulla is a morally bad person] does not exist (and is thus not factively grounded in anything). At $w_{2}$, [Sulla is a morally bad person] exists and is factively grounded in [Sulla takes pleasure in the suffering of others], and [Sulla is a compulsive liar] does not exist. At $w_{3}$, [Sulla is a morally bad person] exists and is factively grounded in [Sulla is a compulsive liar], and [Sulla takes pleasure in the suffering of others] does not exist. This means that, at $w_{1}$, [Sulla is a morally bad person] is non-factively grounded in [Sulla takes pleasure in the suffering of others] (since, at $w_{1}$, there is a possible world where the former fact is factively grounded in the latter). Now consider again the familiar notion of simple foundation:

(Simple Foundation) $y$ dependssF on $x=\mathrm{df}$. Necessarily:

if $y$ exists, then either $y$ is at least partially grounded in $x$, or $y$ is at least partially grounded in some fact about $x$.

(The definiens of Simple Foundation should be read as talking about factive grounding, since Correia (2005, p.65) endorses factivity for grounding.) In $w_{1}$, it is not the case that [Sulla is a morally bad person] depends $\mathrm{SF}$ on [Sulla takes pleasure in the suffering of others], since there are worlds accessible from $w_{1}$ where the former fact exists without being factively grounded in the latter fact (or some fact about the latter fact). Thus, at $w_{1}$ [Sulla is a morally bad person] is non-factively grounded in [Sulla takes pleasure in the suffering of others], without depending ${ }_{\mathrm{SF}}$ on it. In other words: simple foundation is not necessary for non-factive grounding. And the same line of reasoning would seem to apply to all other forms of rigid dependence. ${ }^{29}$ We can therefore conclude that rigid dependence is not necessary for non-factive grounding.

Is it sufficient? It seems that the answer will hinge on the form of rigid dependence in question. Consider again simple foundation. That $y$ depends $\mathrm{SF}$ on $x$ is not sufficient for $y$ to be non-factively grounded in $x$. To see this, suppose that the fact [Caesar is seeing maroon] can only exist at a world by being factively grounded in some fact of the form [Caesar's brain is in state $N$ ] at that world, but that the former fact is never grounded directly in Caesar's brain (that biological object). It is then the case that [Caesar is seeing maroon] depends $\mathrm{SF}_{\mathrm{SF}}$ on Caesar's brain-since the former cannot exist without being factively grounded in some fact about the latter-but it is not the case that [Caesar is seeing maroon] is non-factively grounded in Caesar's brain. In this scenario, there is no possible world where the fact is factively grounded in the brain itself (as opposed to facts about the brain). And since we are assuming that $y$ is non-factively grounded in $x$ if, and only if, $y$ is possibly factively grounded in $x$, it follows that the scenario is one where $y$ depends $\mathrm{SF}$ on $x$ without $y$ being non-factively grounded in $x$.

However, this reasoning does not seem to generalize to all varieties of rigid dependence. Recall e.g. the definition of explanatory existential dependence:

(Explanatory Existential Dependence) $y$ depends EED $_{\text {on }} x=\mathrm{df}$. Necessarily:

(i) if $y$ exists, then $x$ exists; and

(ii) if $y$ exists and $x$ exists, then $y$ exists at least partially because $x$ exists.

29 Considerations of space force me to leave it as an exercise for the reader to verify this. 
(I will assume for the purposes of this discussion that " $y$ exists at least partially because $x$ exists" is equivalent to " $y$ is at least partially factively grounded in $x$." If that assumption is incorrect, we could instead stipulatively introduce a new variety of dependence incorporating the necessary changes, and use that variety for the following discussion.)

Explanatory existential dependence does indeed seem sufficient for non-factive grounding. First, suppose e.g. that $\{$ Caesar $\}$ depends EED $_{\text {on Caesar-in every world }}$ where $\{$ Caesar $\}$ exists, it is at least partially factively grounded in Caesar. Second, suppose that there is at least one world where $\{$ Caesar\} exists. (This second supposition seems very minimal, given the first one. If there is no world where \{Caesar exists, $\{$ Caesar $\}$ is an impossible entity. But the idea of an impossible entity seems highly problematic, and that of an ontologically dependent impossible entity perhaps even more so. In the following, I will assume that if $y$ is ontologically dependent, there is at least one possible world where $y$ exists.) Now consider a world $w_{1}$ where neither $\{$ Caesar $\}$ nor Caesar exists. At $w_{1}$, \{Caesar $\}$ depends EED $_{\text {on Caesar. By our }}$ second supposition, there is a world $w_{2}$ (distinct from $w_{1}$ ) where $\{$ Caesar $\}$ does exist. It follows from our first supposition (and the definition of explanatory existential dependence) that at $w_{2},\{$ Caesar $\}$ is at least partially factively grounded in Caesar. And that means that at $w_{1},\{$ Caesar $\}$ is at least partially non-factively grounded in Caesar. Thus, explanatory existential dependence is sufficient for at least partial non-factive grounding.

So there is at least one variety of ontological dependence that is sufficient for nonfactive grounding. ${ }^{30}$ (I will not consider whether any further varieties are, though they may well be.) Factive and non-factive grounding thus differ in this respect. Why is this? The reason is at least partly that factive and non-factive grounding (at least as we have, following Fine, been thinking about the latter) have different modal profiles. Whether an entity is factively grounded in another at a given world $w$ is what we might call a modally local issue. It hinges only on what goes on at $w$ itself, and other worlds are irrelevant. But whether an entity is non-factively grounded in another at $w$ is a modally non-local issue-what goes on at any possible world (including $w$ itself) can be relevant to that issue. And dependence is modally non-local in this way too. Whether $y$ ontologically depends on $x$ at $w$ does not hinge only on what goes on at $w .{ }^{31}$ This means that the argument employed in Sect. 4 to show that rigid dependence is not sufficient for factive grounding does not necessarily apply when we consider non-factive grounding instead.

Since we have seen that no form of rigid dependence is necessary for non-factive grounding (due to the Flexibility Argument), the modal connection between non-

\footnotetext{
30 Strictly speaking, explanatory existential dependence is only sufficient for at least partial non-factive grounding. But this qualification is fairly unimportant. We could easily stipulatively introduce a new definition of explanatory existential dependence where clause (ii) requires full factive grounding, and then run the same reasoning to arrive at the conclusion that this new variety of dependence is sufficient for full non-factive grounding. I wish to thank an anonymous referee for comments on this point.

31 In fact, questions of dependence should be considered to be not only modally non-local, but indeed modally global, since I've argued that if $y$ depends on $x$ in any possible world, then $y$ depends on $x$ in every possible world. To fix whether $y$ ontologically depends on $x$, the whole of metaphysical space will thus be relevant.
} 
factive grounding and explanatory existential dependence (or any other variety of dependence that might be sufficient for non-factive grounding) only runs in one direction. However, we seem to have identified one interesting connection between non-factive grounding and at least one form of dependence, a connection that is wholly absent in the more commonly considered case of factive grounding.

\section{Generic dependence and the principle of metaphysical insufficiency}

Even though we have now dropped the standard assumption that grounding is factive and investigated a non-factive conception of grounding, we have yet to explore every relevant aspect of the relation between grounding and ontological dependence. For all the varieties of dependence considered so far have been forms of rigid dependence, where the dependent entity $y$ depends on some specific entity $x$. But there are also forms of generic dependence, where an entity $y$ does not depend (or at least need not depend) on some specific entity, but rather on there being some entity or other satisfying the right kind of condition. ${ }^{32}$ Tahko and Lowe (2015) provide the following definition of generic dependence:

(Generic Dependence) $y$ depends $_{\mathrm{G}}$ on $F \mathrm{~s}=\mathrm{df}$. Necessarily, $y$ exists only if some $F$ exists. (Tahko and Lowe 2015, §2)

Various ideas in metaphysics are best cashed out in terms of generic dependence claims. For example, an "Aristotelian" view of universals implies that there cannot be uninstantiated universals. For the property of being a philosopher to exist, for example, there must be at least one philosopher. ${ }^{33}$ So, on this view, the property of being a philosopher depends $\mathrm{G}_{\mathrm{G}}$ on instances of being a philosopher. But the property does not depend on any specific instance. The Aristotelian view is not e.g. that the property of being a philosopher depends on Cicero, even though he is one of its instances. The property can exist without Cicero (as long as it has some other instance). So generic dependence can hold without any corresponding form of rigid dependence.

Before we can consider the relation between grounding and generic dependence, I need to introduce one further component. To get a determinate claim of generic dependence we need some condition to play the role of $F$. And the condition in question will be derived from a promising-looking principle about the modal behavior of grounding:

(Principle of Metaphysical Insufficiency) If $y$ is possibly factively grounded, then, necessarily, if $y$ exists there is some $x$ such that $y$ is factively grounded in $x$.

The Principle of Metaphysical Insufficiency (or PoMI, for short) says that if an entity is factively grounded in one possible world, then it is factively grounded in every world where it exists. Note that it does not follow that the grounded entity has the same factive ground in every world where it exists. The PoMI is perfectly compatible

\footnotetext{
${ }^{32}$ For discussions of the rigid/generic distinction, see e.g. Fine (1995, pp. 287-289), Correia (2008, pp. 1015-1016), and Tahko and Lowe (2015, §2).

33 An influential contemporary proponent of this view is Armstrong (1997).
} 
with cases of flexible grounding as discussed in Sect. 3 above. It only requires that there be some (factive) ground or other for $y$ in every world where $y$ exists.

I will argue that if the PoMI is true, then it entails a connection between grounding and a certain form of generic dependence. But before introducing that form of dependence and discussing its relation to grounding, let us consider the case for and against believing in the PoMI in the first place.

As to the first issue, many paradigmatic examples of grounding seem to fit the principle. For instance, a disjunctive fact like [Snow is purple or Caesar is emperor] can only exist if at least one of [Snow is purple] or [Caesar is emperor] factively grounds it. The disjunctive fact couldn't exist brutely. The same goes for an existentially generalized fact like [There is a philosopher]: to exist it must have some ground, like [Cicero is a philosopher] or [Marcus Aurelius is a philosopher]. And if the truth of the proposition $\langle p\rangle$ is grounded in the truthmaker for $\langle p\rangle$, then the truth of $\langle p\rangle$ must have some factive ground or other. ${ }^{34}$ Finally, it is commonly agreed that particular moral facts_-like the fact [ $a$ is morally right] (where $a$ is a token action) — cannot be brute, but must exist in virtue of the existence of some non-moral fact or other.

In light of such examples, the PoMI has some initial plausibility. But it is not beyond doubt. There is at least one potential counterexample to the principle: mental facts. Even if [Cicero has a headache] is factively grounded in [Cicero is in neural state $N$ ] in the actual world, we might think that the same phenomenal state could have existed as a brute state in a Cartesian substance, without any factive ground whatsoever. For that reason, physicalism is often advanced as a contingent claim. If this is right, the PoMI is false. However, the contingency of physicalism is controversial and has been questioned by Levine \& Trogdon (2009). If they are right, the implication that a grounding claim about the mental commits one to the impossibility of brute mentality is unproblematic.

These are important and difficult questions that deserve more attention than I can currently give them. For our purposes, it will have to suffice to note that the PoMI raises difficult issues in modal metaphysics. It is not clear that the principle will turn out defensible in the end.

Let us now turn to the issue of what the Principle would imply. The truth of the PoMI would entail that a certain form of generic dependence is necessary for (both factive and non-factive) grounding. Consider the following notion:

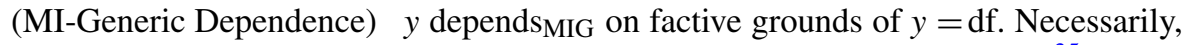
$y$ exists only if some factive ground of $y$ exists. ${ }^{35}$

\footnotetext{
34 It is not uncommon among truthmaker theorists to hold that some true propositions lack truthmakers. But, to the best of my knowledge, no one has held that there are propositions whose truth is grounded in some worlds but brute in others.

35 There are slightly different conceptions of generic dependence available in the literature. Fine (1995) introduces a notion of generic dependence similar to that defined above but with an essentialist operator ("it is true in virtue of the identity of $y$ that") in place of the necessity operator (p. 288). While this Finean notion of generic dependence is perfectly respectable as such, it is not particularly useful for our purposes. I take it that for many grounded entities, it is not part of their essence-part of what it is to be them - that they exist only if they have some ground (even if that same condition might be necessary for them to exist). I wish to thank an anonymous referee for bringing this alternative conception of generic dependence to my attention.
} 
If the PoMI is true, every possibly grounded entity $y$ depends MIG $_{\text {on factive grounds }}$ of $y$. I will start by considering the relation between MI-generic dependence and factive grounding. The former is necessary for the latter, given that the PoMI is true. Suppose that [Sulla is a morally bad person] is actually factively grounded in [Sulla takes pleasure in the suffering of others]. From the PoMI, it then follows that [Sulla is a morally bad person] cannot exist without having some factive ground or other. That is just to say that, necessarily, [Sulla is a morally bad person] exists only if some factive ground of it exists. If the PoMI is true, MI-generic dependence is necessary for factive grounding.

The converse does not, however, seem to hold. At the start of Sect. 4 above, I stated that I will take a form of generic dependence to be sufficient for grounding just in case $y$ 's depending generically in that way on $F$ s at a world $w$ entails the truth of some specific claim of the form " $y$ is grounded in $x$ " at $w$. This is a fairly lax interpretation of sufficiency for generic dependence-we are not even requiring that whatever entity plays the role of $x$ be one of the $F$ s. But even on this liberal requirement, MI-generic dependence is not sufficient for factive grounding. And this is for familiar reasons. Consider the actual world. Here \{Pegasus $\}$ depends ${ }_{\text {MIG }}$ on factive grounds of \{Pegasus $\}$ - the singleton can only exist if it has a factive ground. But since $\{$ Pegasus does not exist in the actual world, \{Pegasus $\}$ is not factively grounded in anything. So MI-generic dependence is not sufficient for factive grounding.

What about non-factive grounding and MI-generic dependence? Since the latter is necessary for factive grounding, it is necessary for non-factive grounding as well. In the actual world, \{Pegasus $\}$ isn't factively grounded in anything. But it is non-factively grounded in Pegasus. And since that implies that there are other possible worlds where \{Pegasus $\}$ is factively grounded in Pegasus, the PoMI guarantees that $\{$ Pegasus $\}$ can only ever exist if some factive ground of it exists. And that dependence claim is true even at the actual world. But that is just to say that in the actual world, \{Pegasus depends $_{\text {MIG }}$ on factive grounds of $\{$ Pegasus $\}$. So MI-generic dependence is necessary for non-factive grounding if the PoMI is true.

The issue of sufficiency will get somewhat more complicated. Again, for MI-generic dependence to be sufficient for (non-factive) grounding it needs to be the case that $y$ 's depending $_{\text {MIG }}$ on factive grounds of $y$ (at a world $w$ ) entails the truth of some specific claim of the form " $y$ is (non-factively) grounded in $x$ " at $w$. But this does not quite seem to be the case. Intuitively speaking, the problem is that an entity $y$ 's depending MIG $_{\text {on }}$ factive grounds of $y$ in $w$ is not a specific enough truth about how $y$ is grounded to entail the requisite specific (non-factive) grounding claims in $w$. In a metaphor: the truths about the MI-generic dependence of $y$ that we are inputting into $w$ do not allow us to output any specific truths of the form " $y$ is grounded in $x$ " at $w$.

This can be shown more precisely via the following reasoning. Start in the actual world, and suppose that [Sulla is a morally bad person] does not exist there. Still, that fact actually depends ${ }_{\text {MIG }}$ on factive grounds of [Sulla is a morally bad person]-in every possible world in which [Sulla is a morally bad person] does exist, it has some factive ground or other. Now, I have assumed that if an entity $y$ is ontologically dependent, there is at least some possible world where $y$ exists. This entails that there is some world $w$, distinct from the actual world, where [Sulla is a morally bad person] does exist. And [Sulla is a morally bad person]'s depending MIG $_{\text {on grounds of [Sulla }}$ 
is a morally bad person] entails that at $w$ [Sulla is a morally bad person] has a factive ground. But nothing in the assumptions made so far entail anything about which specific factive ground [Sulla is a morally bad person] has at $w$. It might be [Sulla takes pleasure in the suffering of others], or it might be [Sulla is a compulsive liar]. As far as our assumptions are concerned it might be some completely different fact—or even some non-fact entity. But if our assumptions so far (including the assumption that, in

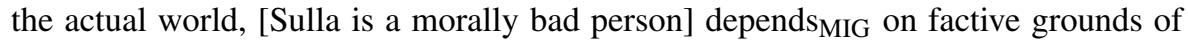
[Sulla is a morally bad person]) do not entail any specific truths of the form "[Sulla is a morally bad person] is factively grounded in $x$ " at $w$, then they do not entail any specific truths about what [Sulla is a morally bad person] is non-factively grounded in at the actual world. For we have assumed, following Kit Fine's suggestion, that $y$ is non-factively grounded in $x$ if, and only if, $y$ is possibly factively grounded in $x$. So MI-generic dependence is not sufficient for non-factive grounding.

There are, however, some qualifications to note in connection with this conclusion. To begin with, even though MI-generic dependence is not sufficient for non-factive grounding (given our demands on sufficiency), it does seem sufficient for something weaker in the vicinity. For if it is the case that there is some world $w$, distinct from the actual world, where [Sulla is a morally bad person] exists and has some factive ground, then we might perhaps say that in the actual world, [Sulla is a morally bad person] is non-factively grounded in something or other. But note that this is quite different from saying that [Sulla is a morally bad person] is non-factively grounded in something specific, like e.g. [Sulla takes pleasure in the suffering of others]. [Sulla is a morally bad person]'s being non-factively grounded in something or other is generic in the sense that it does not amount to the fact in question being related to any specific entity.

Having observed this, we might return to my statement of what it would take for a form of generic dependence to be sufficient for grounding (first formulated in Sect. 4). That statement demands that $y$ 's depending generically in the relevant way on $F$ s at a world $w$ should entail the truth of some specific claim of the form " $y$ is grounded in $x$ " at $w$. In light of what I've just said, one might, however, ask why this demand couldn't be relaxed. Why, more specifically, shouldn't a form of generic dependence count as sufficient for grounding if an entity $y$ 's depending generically in that way on $F$ s at a world $w$ entails not some specific claim of the form " $y$ is grounded in $x$ " at $w$, but rather that $y$ has some ground or other at $w$ ? (As we have noted, this latter proposed criterion is satisfied by MI-generic dependence under a non-factive conception of grounding.)

The best motivation for going for the stricter criterion that I have proposed is, I believe, that it is truer to how grounding has been conceived of in the literature. Even though grounding has increasingly seen both factive and non-factive treatments, it has been construed in both guises as a direct, de re connection between specific entities. (This is part of what is captured by Stipulation 1 of Sect. 2, which takes the canonical form of a grounding claim to be " $y$ is grounded in $x$ ".) From this perspective, MI-generic dependence seems sufficient not for the two-sided de re connection of 
non-factive grounding itself, but rather for at most a property of the dependent entity-namely the property of being non-factively grounded in something or other. ${ }^{36,37}$

It must be admitted, however, that the issue of precisely what we should demand for a form of dependence to count as sufficient for grounding seems to some extent terminological (insofar as some philosophers might be perfectly happy to apply the label "(non-factive) grounding" to what strikes me as more aptly called "the property of being non-factively grounded in something or other"). Perhaps there is even something to be said for introducing a rigid/generic distinction for grounding similar to the one applied to ontological dependence. If so, and if it suffices for generic (non-factive) grounding that there is some entity $x$ such that [Sulla is a morally bad person] is (rigidly) non-factively grounded in $x$, then MI-generic dependence is, after all, sufficient for one form of grounding. This conclusion, however, hinges on this generic conception of grounding being tenable in the end. ${ }^{38}$ Just like philosophers have recently started investigating the possibility and features of non-factive grounding, it may be a worthwhile topic for further research to inquire into the idea of generic grounding. Lack of space forces me to leave the issue at that, noting again that on the more orthodox, non-generic approach to grounding that has standardly been assumed, MI-generic dependence is not sufficient even for non-factive grounding. ${ }^{39}$

\section{Conclusion}

In this article, I have investigated the relation between grounding and ontological dependence through their modal connections (or lack thereof). I argued that on the factive conception of grounding that has been assumed in most of the grounding literature, no form of rigid ontological dependence found in the literature is either necessary or sufficient for grounding. The somewhat poorly delineated nature of ontological dependence makes it difficult to know exactly how far this result generalizes. However, I have tentatively suggested that there are uses of the notion that presuppose

\footnotetext{
36 Since we are supposing in the line of reasoning above that [Sulla is a morally bad person] does not exist in the actual world, this presupposes that an entity which doesn't exist at a world $w$ can still instantiate a property at $w$. This is a presupposition I can personally live with, but it does not seem essential at any rate. Any philosopher who rejects the presupposition can apply whatever strategy she normally employs to capture apparent predicational truths about non-existents.

37 That the holding of MI-generic dependence entails at most a property of the dependent entity should perhaps not come as a surprise. MI-generic dependence is a form of generic dependence, which does not connect two specific entities (but rather "connects" one specific entity with a generic condition). I believe it is for this reason that Correia (2008) writes: "While rigid necessitation [i.e. rigid dependence] is a binary relation, generic necessitation [i.e. generic dependence] of an $F$ (for each particular " $F$ ") is a property." (p. 1015). While I have in Sect. 4 tentatively accepted that ontological dependence is in general not a relation (supposing that relations always relate existent entities), I believe Correia is right in that there is a further reason why generic dependence is not a relation-a reason that does not apply to rigid dependence.

38 Of course, there is little question that if there are facts of the form [ $y$ is grounded in $x$ ] then there are facts of the form [There is some $x$ such that $y$ is grounded in $x$ ]. The potentially interesting question is whether facts of the latter kind can play the right kind of theoretical roles, and are sufficiently similar to facts of the former kind, to warrant thinking of them as belonging to a further variety of grounding.

39 I wish to thank an anonymous referee for comments on the argument.
} 
necessary conditions for ontological dependence that would allow us to generalize the arguments to all possible forms of rigid ontological dependence.

When we broaden the discussion by considering other notions of grounding and ontological dependence (beyond factive grounding and rigid ontological dependence), the picture becomes more complicated. I have argued that while no extant form of rigid dependence is necessary for non-factive grounding, there is at least one form of rigid dependence that is sufficient for it (and it is likely that more examples can be found). I have also argued that given the truth of the Principle of Metaphysical Insufficiency, there is a form of generic ontological dependence that is necessary (though not sufficient) for both factive and non-factive grounding. But it must be emphasized that the Principle is likely to be controversial and that my discussion of it has been extremely cursory. Developing a more principled approach to the difficult question of whether entities that are grounded in one world have to be grounded in every world where they exist would be an interesting and potentially fruitful avenue for further research on grounding.

The preceding discussion shows that a non-factive conception of grounding entails at least some connections with dependence that are absent on a factive conception. Thus far, the notion of non-factive grounding has mostly been explored in formal work on the logic of grounding. One contribution of this article has been to explore differences and similarities between factive and non-factive grounding in one distinctively metaphysical respect, namely in how they relate to ontological dependence. These results suggest that it may be fruitful for metaphysicians to consider whether the move from a factive to a non-factive conception of grounding changes the relations between grounding and other important phenomena in metaphysics too.

Acknowledgements I would like to thank audiences at the "Grounding Gothenburg" workshop at the University of Gothenburg, the "Topics in Ontology and Metaontology" workshop at Uppsala University, research seminars at the University of Helsinki and Umeå University, and the Higher Seminar in Practical Philosophy at Uppsala University. I would particularly like to thank Jan Almäng, Matti Eklund, Sten Lindström, Jon Litland, Donnchadh O'Conaill, Jonathan Shaheen, Alexander Skiles, Andreas Stokke, Tuomas Tahko, Tobias Wilsch, and three anonymous referees for very helpful comments and discussion.

Open Access This article is distributed under the terms of the Creative Commons Attribution 4.0 International License (http://creativecommons.org/licenses/by/4.0/), which permits unrestricted use, distribution, and reproduction in any medium, provided you give appropriate credit to the original author(s) and the source, provide a link to the Creative Commons license, and indicate if changes were made.

\section{References}

Armstrong, D. M. (1997). A world of states of affairs. Cambridge: Cambridge University Press. Baron, S. (2015). The priority of the now. Pacific Philosophical Quarterly, 96(3), 325-348.

Clark, M. J., \& Liggins, D. (2012). Recent work on grounding. Analysis, 72(4), 812-823.

Correia, F. (2005). Existential dependence and cognate notions. Munich: Philosophia.

Correia, F. (2008). Ontological dependence. Philosophy Compass, 3(5), 1013-1032.

Correia, F. (2014). Logical grounds. The Review of Symbolic Logic, 7(1), 31-59.

Correia, F., \& Schnieder, B. (2012). Grounding: An opinionated introduction. In F. Correia \& B. Schnieder (Eds.), Metaphysical grounding: Understanding the structure of reality (pp. 1-36). Cambridge: Cambridge University Press.

Dasgupta, S. (2014). The possibility of physicalism. The Journal of Philosophy, 111(9), 557-592.

Dasgupta, S. (2016). Metaphysical rationalism. Noûs, 50(2), 379-418. 
Fine, K. (1994). Essence and modality. Philosophical Perspectives, 8, 1-16.

Fine, K. (1995). Ontological dependence. Proceedings of the Aristotelian Society, 95, 269-290.

Fine, K. (2012). Guide to ground. In F. Correia \& B. Schnieder (Eds.), Metaphysical grounding: Understanding the structure of reality (pp. 37-80). Cambridge: Cambridge University Press.

Jenkins, C. S. (2011). Is metaphysical dependence irreflexive? The Monist, 4(2), 267-276.

Kment, B. (2014). Modality and explanatory reasoning. Oxford: Oxford University Press.

Levine, J., \& Trogdon, K. (2009). The modal status of materialism. Philosophical Studies, 145(3), 351-362.

Lewis, D. K. (1986). On the plurality of worlds. Oxford: Basil Blackwell.

Litland, J. E. (2017). Grounding ground. In K. Bennett \& D. Zimmerman (Eds.), Oxford studies in metaphysics (Vol. 10, pp. 279-316). Oxford: Oxford University Press.

Lowe, E. J. (1998). The possibility of metaphysics. Oxford: Oxford University Press.

Mulligan, K., Simons, P., \& Smith, B. (1984). Truth-makers. Philosophy and Phenomenological Research, 44(3), 287-321.

Raven, M. J. (2013). Is ground a strict partial order? American Philosophical Quarterly, 50(2), 191-199.

Rosen, G. (2010). Metaphysical dependence: Grounding and reduction. In B. Hale \& A. Hoffmann (Eds.), Modality: Metaphysics, logic, and epistemology (pp. 109-135). Oxford: Oxford University Press.

Schaffer, J. (2008). Truth and fundamentality: On Merricks's Truth and ontology. Philosophical Books, 49(4), 302-316.

Schaffer, J. (2009). On what grounds what. In D. Chalmers, D. Manley, \& R. Wasserman (Eds.), Metametaphysics (pp. 347-383). Oxford: Oxford University Press.

Schaffer, J. (2010). Monism: The priority of the whole. Philosophical Review, 119(1), 31-76.

Schaffer, J. (2012). Grounding, transitivity, and contrastivity. In F. Correia \& B. Schnieder (Eds.), Metaphysical grounding: Understanding the structure of reality (pp. 122-138). Cambridge: Cambridge University Press.

Schaffer, J. (2016). Grounding in the image of causation. Philosophical Studies, 173(1), 49-100.

Schnieder, B. (2006). A certain kind of trinity: Dependence, substance, explanation. Philosophical Studies, 129(2), 393-419.

Schnieder, B. (2017). Grounding and dependence. Synthese. https://doi.org/10.1007/s11229-017-1378-z.

Skiles, A. (2015). Against grounding necessitarianism. Erkenntnis, 80(4), 717-751.

Tahko, T. (2015). An introduction to metametaphysics. Cambridge: Cambridge University Press.

Tahko, T. E., \& Lowe, E. J. (2015). Ontological dependence. In E. N. Zalta (Ed.), The Stanford encyclopedia of philosophy (Spring 2015 edition). http://plato.stanford.edu/entries/dependence-ontological/.

Trogdon, K. (2013). An introduction to grounding. In M. Hoeltje, B. Schnieder, \& A. Steinberg (Eds.), Varieties of dependence-Ontological dependence, grounding, supervenience, response-dependence (pp. 97-122). Munich: Philosophia.

Wilsch, T. (2015). The deductive-nomological account of metaphysical explanation. Australasian Journal of Philosophy, 94(1), 1-23.

Wilson, J. (2017). Determinables and determinates. In E. N. Zalta (Ed.), The Stanford encyclopedia of philosophy (Spring 2017 edition). https://plato.stanford.edu/archives/spr2017/entries/determinate-de terminables/.

Witmer, D. G., Butchard, W., \& Trogdon, K. (2005). Intrinsicality without naturalness. Philosophy and Phenomenological Research, 70(2), 326-350. 